\title{
Multidimensional Potential Energy Surfaces
}

\section{resolved at the RASPT2 level for accurate photoinduced isomerization dynamics of}

\section{azobenzene}

\author{
Flavia Aleotti, ${ }^{\dagger, \ddagger}$ Lorenzo Soprani, ${ }^{\dagger, \dagger}$ Artur Nenov, ${ }^{*}$, Roberto Berardi, $^{\dagger}$ Alberto \\ Arcioni $^{\dagger}{ }^{\text {Claudio Zannoni, }}{ }^{\dagger}$ and Marco Garavelli*,† \\ $\dagger$ †ipartimento di Chimica Industriale "Toso Montanari", Universitá di Bologna, Viale del \\ Risorgimento 4, 40136 Bologna, Italy \\ $\ddagger$ This authors contributed equally to this work \\ E-mail: artur.nenov@unibo.it; marco.garavelli@unibo.it
}

\begin{abstract}
We have used state-of-the-art ab initio RASPT2 computations using a 16 orbitals, 18 electrons active space to produce an extended three dimensional map of the potential energy surfaces (PESs) of the ground and first $n \pi^{*}$ excited state of azobenzene along CNNC torsion and the two CNN bending angles, which are the most relevant coordinates for the trans-cis photoisomerization process. Through comparison with fully unconstrained optimizations performed at the same level of theory, we show that the three selected coordinates suffice to correctly describe the photoisomerization mechanism and the $S_{1}-S_{0}$ crossing seam. We also provide a map of the non-adiabatic coupling between the two states in the region where they get closer in energy. Eventually, we
\end{abstract}


show that treating the two CNN bending angles as independent coordinates is fundamental to break the symmetry and couple the two electronic states. The accuracy of the $S_{0}$ and $S_{1}$ PESs and couplings was validated with semiclassical dynamics simulations in the reduced space of the scanned coordinates, showing results in good agreement with published full-coordinates dynamics.

\section{Introduction}

Azobenzene and its derivatives are well known for their ability to undergo a significant and ultrafast structural change when irradiated with light (photoisomerization). This change in the molecular geometry on going from trans to cis or vice versa, together with other favorable properties such as the reversibility of the photoisomerization process, the stability of both isomers and the photoactivity even under unfavorable or constrained conditions, make azobenzene the perfect candidate for application in light-powered nanomachines, actuators ${ }^{1,2}$ and in general in materials with photomodulable properties. ${ }^{3-9}$ Even though azobenzene is quite a simple molecule, the understanding of its trans-cis interconversion mechanism has been challenging chemists for over eighty years. In particular, a pronounced dependence of the photoisomerization quantum yield on the excitation wavelength has been documented, ${ }^{10,11}$ which is in violation of Vavilov's extension of Kasha's rule (also known as the Kasha-Vavilov's rule). This dependence suggests that different mechanisms take place when the system is irradiated with visible light (excitation to $S_{1}, n \pi^{*}$ in nature) or with UV light (excitation to $S_{2}, \pi \pi^{*}$ in nature). The application of highly accurate (CASSCF/CASPT2) potential energy

surfaces (PESs) mapping ${ }^{11-13}$ has given a clear idea of the possible excited state deactivation routes. Indeed, there are two limit mechanisms for the photoconversion between the trans and cis isomers: pure torsion around the $\mathrm{N}=\mathrm{N}$ bond or pure in-plane inversion of one of the two CNN angles, and the relevance of each of these pathways seems to depend on the starting state. According to the latest computational works, ${ }^{11,12,14}$ on the ground state (thermal reaction) a huge barrier is observed along torsion, while the potential energy surface (PES) 
of $S_{1}$ is rather flat along the same coordinate, but displays a considerable barrier along the inversion pathway. Several crossing points (conical intersections, or CIs) between $S_{2}, S_{1}$ and $S_{0}$ have been found along both torsional and inversion photoisomerization pathways, ${ }^{11-13,15}$ but the most likely scenario is that there is a wide crossing seam, which is reached via a combination of CNNC torsion and $\mathrm{CNN} / \mathrm{NNC}$ bending. ${ }^{11,15}$ Given this picture, excited state dynamic simulations become fundamental to understand what happens after the $n \pi^{*}$ or $\pi \pi^{*}$ states are populated, as well as to compare the calculated quantum yields with the experimental ones to validate the model picture itself. Excited state dynamics in a polyatomic system like azobenzene requires some approximations: this means either to perform a full quantum dynamics (QD) but in a reduced-coordinate space (i.e., considering only the reactive coordinates), or to perform dynamics in the full space of nuclear coordinates but with an approximated method. The latter approach includes both on-the-fly mixed quantum-classical dynamics (e.g., using Tully's trajectory surface hopping algorithm) and classical molecular dynamics (MD) simulations using ad hoc forsce fields parameterized with respect to quantum PESs. Semi-classical dynamics simulations of azobenzene performed by Persico and coworkers ${ }^{15}$ starting from the lowest excited state $\left(n \pi^{*}\right)$ suggested that if the non-adiabatic event takes place much before the CNNC torsion reaches $90^{\circ}$, the trajectory is more likely to be unreactive, leading to the starting $S_{0}$ minimum (i.e., no isomerization). More recent dynamics simulations of azobenzene ${ }^{11}$ confirm this picture, and give a possible explanation to the violation of Kasha's rule: after direct excitation to $S_{1}\left(n \pi^{*}\right)$ the preferred pathway is the torsional one, which is more favorable to isomerization. On the other hand, excitation with UV light to $S_{2}\left(\pi \pi^{*}\right)$ results in a considerable excitation of the in-plane symmetric bending mode, leading to a cascade of $S_{2} / S_{1} / S_{0}$ decay before torsion is activated (non-reactive mechanism).

With this computational work we report the PESs of $S_{0}$ and $S_{1}\left(n \pi^{*}\right)$ of azobenzene in the 3D space spanned by CNNC torsion and the two CNN bending angles (rigid scan) at SS- and MS-RASPT2 level of theory, using a full $\pi$ active space plus the two non-bonding 
$n$ orbitals. To the best of our knowledge, there is no other example in the literature of such an accurate scan including symmetry-breaking coordinates, which is necessary to correctly describe the crossing region. Through comparison with fully-relaxed excited state geometries (minima and CIs obtained at the same level of theory) we also show that these degrees of freedom are sufficient to accurately locate the crossing points where the non-adiabatic decay takes place, and that other coordinates (e.g., NN and CN stretching) are only of secondary importance. The accuracy of the PESs has been validated with semi-classical dynamics in the reduced space of the three torsional and bending coordinates, whose results are in good agreement with past simulations. ${ }^{15-17}$

The reported data lay a solid foundation for further more accurate dynamics calculations using different approaches: both full quantum dynamics in the subspace of the reactive coordinates, or full-space classical and semi-classical MD using an ad hoc parameterized

force field and a proper surface hopping algorithm. Concerning the non-adiabatic event, we have mapped the non-adiabatic coupling (NAC) vector in an extended region of the CNNC dihedral ranging from $80^{\circ}$ to $100^{\circ}$ where the two states get close and strongly interact with each other. Like the PESs, also the calculated NACs can be useful both for QD and for ad hoc schemes in classical and semi-classical simulations (e.g., Tully's surface hopping algorithm ${ }^{18}$ ), ensuring that non-adiabatic events are treated on an equal footing, thus allowing for direct comparison between the results of the different approaches.

\section{Computational methods}

\subsection{Ab initio calculations}

The PESs of both ground and first excited states of azobenzene were mapped along three internal coordinates: the torsional angle around the $\mathrm{N}=\mathrm{N}$ central bond and the two CNN angles. Indeed, the interconversion between the trans and cis isomers can take place either via torsion around the $\mathrm{N}=\mathrm{N}$ bond or via inversion of either $\mathrm{CNN}$ angle, and the wide liter- 
ature on the isomerization process is mainly focused on the relative relevance of these two pathways under different conditions. ${ }^{11-13,19-21}$

A 16 orbitals/18 electrons active space was used for all calculations, including all $\pi$ and $\pi^{*}$ orbitals and also the two non-bonding $n$ orbitals (see supporting information), together with a ANO-L-VDZP basis set. ${ }^{22}$ In all cases, a State-Averaged Restricted Active Space Self Consistent Field (SA-RASSCF) calculation (in which the $\pi$ and $n$ orbitals were put in RAS1, and the $\pi^{*}$ orbitals in RAS3, allowing for maximally four holes in RAS1 and four electrons in RAS3) was followed by a RASPT2 correction, either at the Single State (SS) or at the Multi State (MS) level (see below), using an imaginary shift of 0.2 a.u. and setting the IPEA shift to zero. The quality of the results was assessed by checking the reference wavefunction weight after the perturbation correction, which was always around 60\%. All the energy computations have been performed with the MOLCAS ${ }^{23}$ quantum chemistry program, applying Cholesky decomposition.

The selected torsional and bending coordinates were scanned starting from the MP2/ANOL-VDZP ground-state-optimized structures of both trans- and cis-azobenzene isolated isomers in gas phase (Figure 1). All remaining degrees of freedom were kept frozen at their ground state minimum value (rigid scan). Trans-azobenzene has a $C_{2 h}$ symmetry in its minimum, but the $\sigma_{h}$ plane is removed by torsion around the CNNC dihedral, reducing the symmetry to $C_{2}$. On the other hand, cis-azobenzene already belongs to the $C_{2}$ point group at its $S_{0}$ minimum. For both isomers, the $C_{2}$ axis is preserved under symmetric bending, while it is removed by asymmetric bending deformations, resulting in a $C_{1}$ structure. As a consequence, the structures of 3D scan characterized by identical CNN/NNC bending angles have $C_{2}$ symmetry, while all other geometries have $C_{1}$ symmetry. The results of the two possible rigid scans (i.e., starting from trans or cis ground state equilibrium structure) were eventually merged keeping the structure which gave the lowest $S_{1}$ energy for each point of the $3 \mathrm{D}$ grid.

First, we did a $2 \mathrm{D}$ scan of $\mathrm{CNNC}$ torsion (between $0^{\circ}$ and $180^{\circ}$, step $10^{\circ}$ ) and $\mathrm{CNN} / \mathrm{NNC}$ 


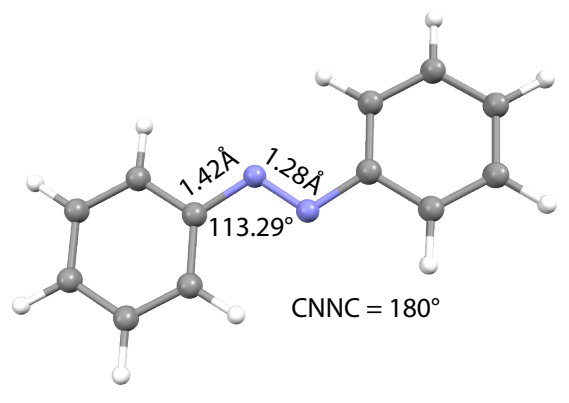

(a) trans-azobenzene

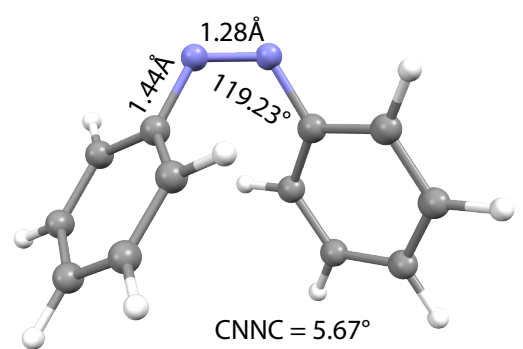

(b) cis-azobenzene

Figure 1: Azobenzene ground state minima (gas phase, ANO-L-VDZP/MP2)

symmetric bending (between $100^{\circ}$ and $160^{\circ}$, step $2^{\circ}$ ). Then, for selected values of the CNNC dihedral (namely $180^{\circ}, 150^{\circ}, 120^{\circ}, 102^{\circ}, 98^{\circ}, 94^{\circ}, 90^{\circ}, 86^{\circ}, 82^{\circ}, 60^{\circ}, 30^{\circ}, 10^{\circ}$ ) we additionally scanned the two CNN bending angles independently, using the same range and step as before, giving rise to roughly 10000 points in the 3D surface.

Benchmarking has shown that the SS- and MS-RASPT2 methods are almost equivalent when $S_{0}$ and $S_{1}$ are well separated in energy, while the mixing around $90^{\circ}$ leads to discontinuities whose behavior is analyzed in detail in the suppotin information; MS was eventually chosen for $82^{\circ} \leq \mathrm{CNNC} \leq 102^{\circ}$, after close analysis of the wavefunction mixing. In all the other cases, the perturbation correction was of the SS-RASPT2 type.

For the purpose of studying the dynamics initiated in the $n \pi^{*}$ state $\left(S_{1}\right.$ in all the points of the $3 \mathrm{D}$ grid), it suffices to include only the first two states in the state averaging procedure (SA2-RASSCF/RASPT2). On the other hand, the $\pi \pi^{*}$ state is the third state $\left(S_{2}\right)$ at the trans Franck-Condon (FC) point, but it exhibits pronounced energetic destabilization for values of the CNNC torsion away from $180^{\circ}$ or symmetric $\mathrm{CNN} / \mathrm{NNC}$ bending above $120^{\circ}$. As a consequence, a large number of states would have to be included in the SARASSCF calculations to keep track of this particular state along the scan, resulting in a high computational cost. For this reason, the $\pi \pi^{*}$ state was mapped only in a small region near the FC point using SS-RASSCF/SA-6-RASPT2 $\left(180^{\circ} \leq \mathrm{CNNC} \leq 120^{\circ}\right.$ and $\mathrm{CNN}$ $=\mathrm{NNC}$ between $100^{\circ}$ and $120^{\circ}$ approximately, see Figure 2 (a) and (b)). This choice is supported by recent results ${ }^{11}$ showing that the excitation to the $\pi \pi^{*}$ state injects energy in 
the symmetric bending mode, while keeping the molecule planar. Then, the steep gradient along symmetric bending causes a considerable decrease of the two CNN/NNC angles, and drives the system towards a planar conical intersection between $S_{2}\left(\pi \pi^{*}\right)$ and $S_{1}\left(n \pi^{*}\right)$, which transfers population to the lowest excited state. It is therefore reasonable to think that, for the study of the reaction mechanism starting from the $\pi \pi^{*}$ state, it should be sufficient to map the surface of $S_{2}$ only close to $\mathrm{CNNC}=180^{\circ}$ and for small values of the two CNN angles (i.e., in a region that goes from the FC point up to the $S_{2} / S_{1} \mathrm{CI}$ ). On the other hand, in the construction of the $3 \mathrm{D}$ grid, the $S_{0}$ and $S_{1}$ energies were calculated including only two states in the state averaging procedure.

The same level of theory of the rigid scans (MS-2-RASPT2/SA-2-RASSCF $(4,9|0,0| 4,7) /$ ANOL-VDZP) was used also for a fully relaxed geometry optimization of the first excited state $\left(n \pi^{*}\right)$ and for a CI optimization, both performed without imposing any symmetry.

Besides the potential energy, near the crossing region, we also mapped the Non-Adiabatic Coupling (NAC) between the involved states, in order to visualize and quantify the degree of wavefunction mixing. The NAC vector was computed at MS-RASPT2 level for the points of our $3 \mathrm{D}$ grid where $82^{\circ} \leq \mathrm{CNNC} \leq 102^{\circ}$ and the energy gap $\Delta E_{S_{1}-S_{0}}$ was lower than $0.3 \mathrm{eV}$. Analytical NACs at the MS-RASPT2 level are not available in MOLCAS, yet their computation can be performed numerically by making a small displacement along every degree of freedom and computing the wavefunction overlap with the reference point using the formula: 24

$$
\mathrm{NAC}_{i j}=\frac{\left(\left\langle\Psi_{i}(r) \mid \Psi_{j}(r+d r)\right\rangle-\left\langle\Psi_{i}(r) \mid \Psi_{j}(r-d r)\right\rangle\right)+\left(\left\langle\Psi_{j}(r) \mid \Psi_{i}(r+d r)\right\rangle-\left\langle\Psi_{j}(r) \mid \Psi_{i}(r-d r)\right\rangle\right)}{4 d r}
$$

A computation of the full NAC would be too expensive, as it would require 144 singlepoint calculations for each geometry in the case of azobenzene. However, from a preliminary analysis of the full NAC vector at some test geometries, we noticed that only the components that refer to the four central C-N-N-C atoms adopt significant values. Given this result, the NAC was calculated only in the subspace of the four central CNNC atoms (24 single-point 
calculations per geometry). Details about the full-coordinate NAC calculations are provided in the supporting information.

Minima and CI optimization with numerical RASPT2 gradients were performed using the suite COBRAMM ${ }^{23-25}$ interfaced with the ab initio software package MOLCAS with an integrated scheme for obtaining numerical gradients and non-adiabatic couplings.

\subsection{Semi-classical dynamics}

The accuracy of the 3D PES was assessed with semi-classical dynamics simulations. The nuclei were propagated classically in the space of CNNC torsion and CNN/NNC bending (i.e., fitting the computed potential energy with a combination of the first 6 terms of the cosine Fourier series for the CNNC dihedral, and the first 6 terms of the power series expansion for the CNN and NNC angles). All the remaining degrees of freedom were effectively frozen by setting the corresponding force field constant to artificially high values. Electrostatic charges and Lennard-Jones coefficients were set to zero as well. The fitting procedure smooths the surface, eliminating any discontinuity or ridge created by the merging of the trans and cis rigid scans (see the supporting information for the fitted PESs).

We ran 20010 ps NVE simulations of a single trans-azobenzene molecule in vacuum at $300 \mathrm{~K}$ with a timestep of $0.1 \mathrm{fs}$. The simulations were started in the first $n \pi^{*}$ excited state, sampling the 3D phase space from a Wigner distribution in the reduced coordinate space around the ground state trans minimum at $300 \mathrm{~K}$.

Whenever the classical trajectory on the excited state entered a region of coupling between the two states (i.e., for $82^{\circ} \leq \mathrm{CNNC} \leq 102^{\circ}$ ), it was allowed to instantaneously hop to the ground state according to Tully's fewest switches probability. ${ }^{18}$ The probability was calculated at every timestep using the standard formula implemented in COBRAMM ${ }^{24}$ in which the NAC value was taken from the nearest geometry from our grid and the atomic velocities were rescaled by a 4.5 factor, that was obtained comparing them to those of a full-coordinate system (see supporting information). 
After the non-adiabatic event, the force field felt by the molecule was changed from that of $S_{1}$ to that of $S_{0}$ until the end of the simulation without allowing for back hopping, since the gradient on the $S_{0}$ surface quickly drives the trajectory out of the crossing region.

All MD simulations were performed with an in-house-modified version of the Molecular Dynamics software NAMD, ${ }^{26}$ while the hopping probability was calculated using the COBRAMM code ${ }^{23-25}$ to propagate the population of the electronic states along the classical trajectory.

\section{Results and discussion}

\subsection{D surface in space of torsion and symmetric bending}

Table 1: Structures and energies* of $S_{0}, S_{1}$ and $S_{2}$ at significant points (minima, saddle points, relevant CIs) obtained with the rigid scan (in the space of CNNC torsion and CNN/NNC bending) and with unconstrained optimizations. The label TXX-Sy refers to the degree of torsion (XX) in state y. ( ${ }^{*} a=\mathrm{SS}-\mathrm{RASPT} 2$, $b=$ MS-RASPT2)

\begin{tabular}{|c|c|c|c|c|c|c|c|}
\hline & & $\mathrm{CNNC}\left({ }^{\circ}\right)$ & $\mathrm{CNN}\left({ }^{\circ}\right)$ & $\mathrm{NNC}\left({ }^{\circ}\right)$ & $E_{S_{0}}(\mathrm{eV})$ & $E_{S_{1}}(\mathrm{eV})$ & $E_{S_{2}}(\mathrm{eV})$ \\
\hline \multirow{9}{*}{$\begin{array}{l}\text { rigid scan of } \\
\mathrm{CNNC} \\
\mathrm{CNN}=\mathrm{NNC}\end{array}$} & $S_{0}$ trans $\min$ & 180 & $112-114$ & $112-114$ & $0.00^{a}$ & $2.66^{a}$ & $3.86^{a}$ \\
\hline & $S_{0}$ cis $\min$ & 0 & 120 & 120 & $0.37^{a}$ & $3.15^{a}$ & - \\
\hline & $S_{0} \mathrm{TS}$ & 90 & 118 & 118 & $1.57^{b}$ & $2.45^{b}$ & - \\
\hline & $S_{1}$ trans $\min$ & 180 & 126 & 126 & $0.51^{a}$ & $2.21^{a}$ & - \\
\hline & $S_{1}$ cis $\min$ & 0 & 126 & 126 & $0.53^{a}$ & $3.03^{a}$ & - \\
\hline & $S_{1}$ symm min & 100 & 120 & 120 & $1.55^{b}$ & $2.22^{b}$ & - \\
\hline & $S_{2} \min$ & 180 & $108-110$ & $108-110$ & $0.11^{a}$ & $2.99^{a}$ & $3.84^{a}$ \\
\hline & $S_{1} / S_{0}$ CI-bend1 & 180 & 150 & 150 & $3.04^{a}$ & $3.08^{a}$ & - \\
\hline & $S_{1} / S_{0}$ CI-bend 2 & 0 & 156 & 156 & $3.22^{a}$ & $3.24^{a}$ & - \\
\hline \multirow{8}{*}{$\begin{array}{l}\text { rigid scan of } \\
\text { CNN, NNC }\end{array}$} & T98- $S_{1} \min$ & 98 & 116 & 134 & $1.79^{b}$ & $2.16^{b}$ & - \\
\hline & T94- $S_{1} \min$ & 94 & 116 & 132 & $1.74^{b}$ & $2.16^{b}$ & - \\
\hline & T90- $S_{1} \min$ & 90 & 116 & 132 & $1.73^{b}$ & $2.16^{b}$ & - \\
\hline & T98- $S_{1} / S_{0}$ CI & 98 & 116 & 146 & $2.12^{b}$ & $2.22^{b}$ & - \\
\hline & T94- $S_{1} / S_{0}$ CI & 94 & 114 & 146 & $2.16^{b}$ & $2.19^{b}$ & - \\
\hline & T90- $S_{1} / S_{0}$ CI & 90 & 114 & 146 & $2.13^{b}$ & $2.20^{b}$ & - \\
\hline & opt- $S_{1} \min$ & 96 & 115 & 145 & $1.98^{b}$ & $1.98^{b}$ & - \\
\hline & opt- $S_{1} / S_{0}$ CI & 95 & 115 & 146 & $1.98^{b}$ & $1.99^{b}$ & - \\
\hline
\end{tabular}

Figure 2 (a) and (b) shows some sections of the 3D PESs of $S_{0}, S_{1}$ and $S_{2}$, as a function of $\mathrm{CNNC}$ torsion and symmetric $\mathrm{CNN} / \mathrm{NNC}$ bending angles (ridges on the surfaces are 


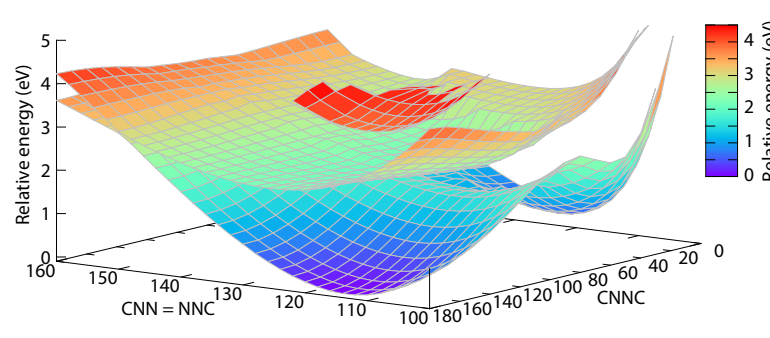

(a)

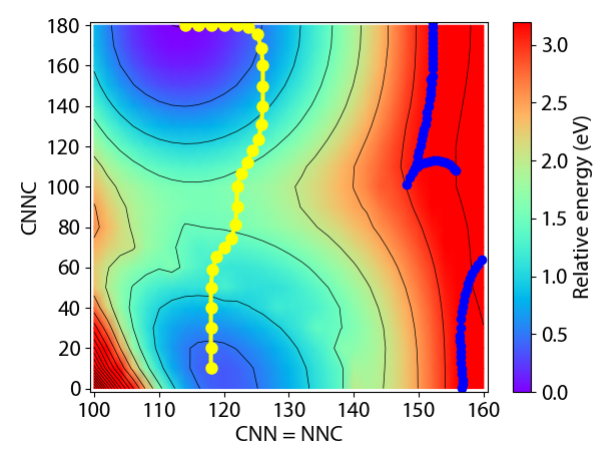

(c)

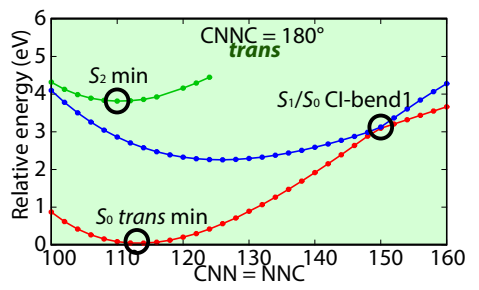

(e)

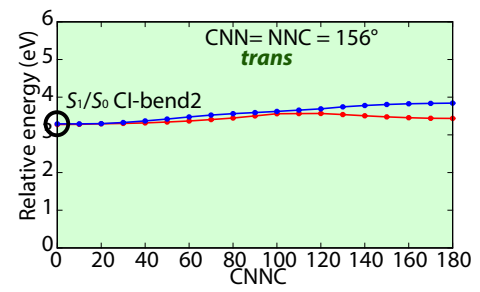

(h)

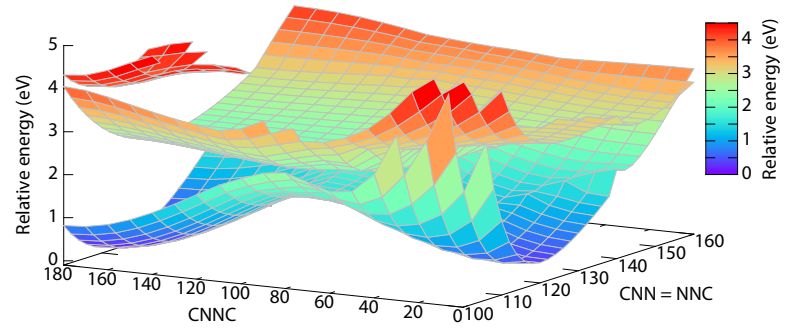

(b)

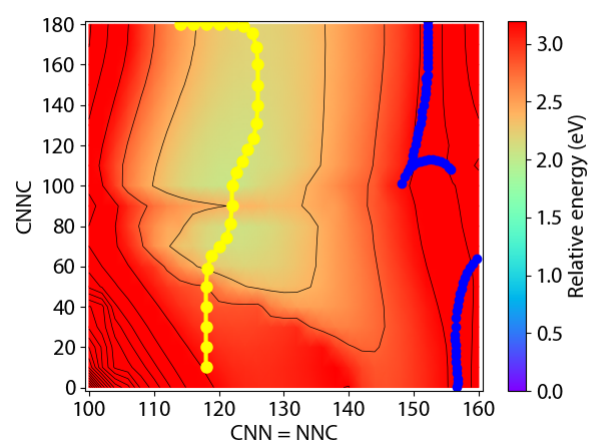

(d)

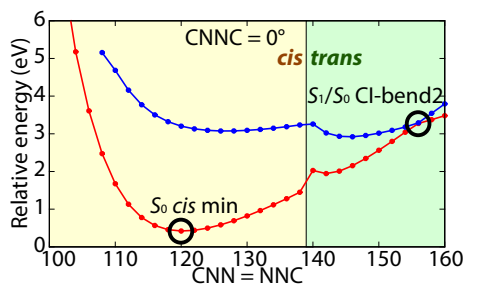

(g)

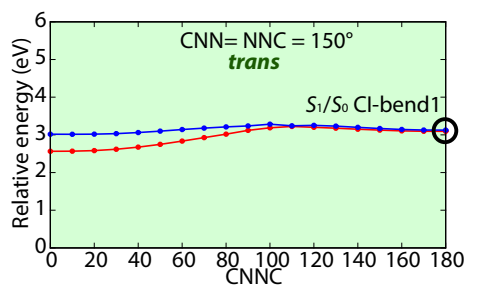

(i)

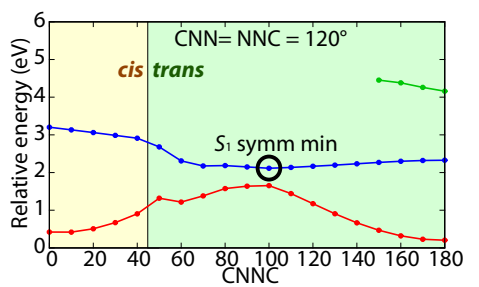

(j)

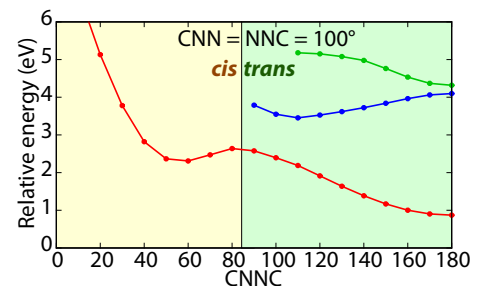

(k)

Figure 2: Side ((a) and (b)) and top views $\left(S_{0}(\mathrm{c})\right.$ and $\left.S_{1}(\mathrm{~d})\right)$ of the PESs in the space of CNNC torsion and symmetric CNN bending; cuts for a selected torsional (e)-(g) and symmetric bending (h)-(k) values $\left(S_{0}\right.$ in red; $S_{1}$ in blue; $S_{2}$ in green, if present). In (c) and (d) the yellow and blue lines represent the minimumenergy path (MEP) on the $S_{1}$ surface from FC points to $S_{1}$ minimum and the CI seam, respectively. In (e)-(k) the region computed for the cis-and trans-derived conformations are marked with yellow and green background, respectively. 
originated by the sudden change in the geometry when passing from trans- to cis-derived structures, as described computational methods section). Details about minima, saddle points and relevant CIs are given in the upper part of Table 1. The vertical excitation to $S_{1}$ is found to be $2.66 \mathrm{eV}$ (466 nm, $436 \mathrm{~nm}$ experimental ${ }^{27}$ ) for $S_{0}$ trans minimum and $2.78 \mathrm{eV}\left(445 \mathrm{~nm}, 436 \mathrm{~nm}\right.$ experimental $\left.{ }^{27}\right)$ for the $S_{0}$ cis minimum, while for $S_{2}$ we only have the trans excitation energy, which is $3.86 \mathrm{eV}\left(321 \mathrm{~nm}, 313 \mathrm{~nm}\right.$ experimental $\left.{ }^{27}\right)$. The small underestimation $(\approx 0.10 \mathrm{eV})$ in the case of the $n \pi^{*}$ state is due to the neglect of solvation effects, which are known to blue-shift the $n \pi^{*}$ transitions, while the $\pi \pi^{*}$ ones are much less affected. ${ }^{28}$ Moreover, in the case of the $n \pi^{*}$ band, the experimental value is not exactly comparable with the calculated value, which refers to the vertical transition: indeed, the $n \pi^{*}$ state is dark, and can only absorb thanks to symmetry-breaking deformations, which could affect the position of the absorption maximum. In spite of all these phenomena, the obtained error is well within the RASPT2 error for the energy calculations $(\approx 0.2 \mathrm{eV})$.

The surface of $S_{0}$ shows a huge torsional barrier separating the two minima, the saddle point $\left(S_{0}\right.$ TS in Table 1$)$ standing $1.57 \mathrm{eV}$ above the trans minimum and $1.20 \mathrm{eV}$ above the cis minimum.

On the other hand, the $S_{1}$ surface along the CNNC torsion is extremely flat, and does not display any barrier within our gridpoints, neither starting from the trans nor starting from the cis isomer. Although it is possible that we may overlook the presence of a torsional saddle point due to the lack of full geometry relaxation, the topology of the $n \pi^{*}$ PES suggests that (if it exists) the barrier should be extremely low, in agreement with past computational studies ${ }^{11,12,14}$ and photoelectron spectroscopy. ${ }^{29}$ As a consequence, the structures $S_{1}$ trans min and $S_{1}$ cis min reported in Table 1 must be considered as relative to the bending coordinate starting from the FC points, while the absolute minimum on $S_{1}$ in this subspace $\left(S_{1}\right.$ symm min in Table 1$)$ is found at $\mathrm{CNNC}=100^{\circ}$. The minimum energy path (MEP) on $S_{1}$ in the subspace of CNNC torsion and symmetric CNN/NNC bending is represented in the left part of Figure 3. On the trans side, starting from FC point $\left(E_{S_{1}}=2.66 \mathrm{eV}\right)$, the 
MEP initially follows the symmetric bending coordinate, up to the $S_{1}$ trans minimum $\left(E_{S_{1}}\right.$ $=2.21 \mathrm{eV})$, then it deviates along torsion, up to the twisted $S_{1}$ symmetric minimum $\left(E_{S_{1}}\right.$ $=2.06 \mathrm{eV})$. The initial gradient along symmetric bending is due to the significant difference in the $\mathrm{CNN} / \mathrm{NNC}$ values between the $\mathrm{FC}$ point and the $n \pi^{*}$ minimum (see Table 1 ) and it is responsible for much of the stabilization on $S_{1}$, while the points of the MEP that go from the planar $S_{1}$ trans minimum up to the twisted $S_{1}$ symmetric minimum are almost isoenergetic. In contrast, on the cis side, the difference in symmetric bending between the FC point and the $S_{1}$ minimum is small, and the MEP is mainly torsional, and characterized by a steeper gradient, since the $\operatorname{cis} \mathrm{FC}$ point $\left(E_{S_{1}}=3.15 \mathrm{eV}\right)$ is higher in energy compared to the trans one. In any case, the MEP on the $n \pi^{*}$ state in this $2 \mathrm{D}$ subspace is mainly torsional, and it lays quite far from the crossing seam (blue line in Figure 2 (c) and (d)). Previous computations on azobenzene ${ }^{11-13,15}$ have found some twisted, low-energy $S_{1} / S_{0}$ CIs but, in our torsion-symmetric bending coordinate space, the molecule cannot desymmetryze, and we still observe a finite $S_{1}-S_{0}$ gap $(0.46 \mathrm{eV})$ at the $n \pi^{*}$ state twisted minimum (see Figure $2(\mathrm{j}))$. In order to find a low-energy CI, a fully-relaxed optimization is needed (see below). Indeed, the $S_{1} / S_{0}$ crossing region revealed in this bidimensional scan only includes high in energy structures $(\approx 3 \mathrm{eV})$ characterized by wide CNN/NNC angles. The two points labelled $S_{1} / S_{0}$ CI-bend1 and $S_{1} / S_{0}$ CI-bend2 (Figures 2 and 3 and Table 1 ) represent the projection of this crossing seam on the symmetric bending coordinate starting from the trans or the cis FC points, respectively. Both these crossing points are sloped CIs (see Figure 2 (e) and (g)) and their presence had already been reported. ${ }^{11-13} S_{1} / S_{0}$ CI-bend1 on the trans side is difficult to reach at room temperature with direct $n \pi^{*}$ excitation, since it lies significantly higher in energy than the trans FC point and the planar $S_{1}$ trans minimum. According to reference, ${ }^{11}$ this crossing point becomes accessible via excitation to the $\pi \pi^{*}$ state, and it is responsible for the violation of Kasha's rule, as explained above. In contrast, on the cis side the FC point and $S_{1} / S_{0}$ CI-bend2 are nearly isoenergetic, but here the gradient on $S_{1}$ is much more favorable to CNNC torsion than to symmetric bending (as shown by the MEP). 
The $S_{2}$ PES is visible only in a small region near the trans FC point (Figure 2), the $\pi \pi^{*}$ minimum from our grid $\left(S_{2}\right.$ min in Table 1$)$ is planar and characterized by a smaller symmetric bending value $\left(\approx 108^{\circ}\right)$, compared to the trans $\mathrm{FC}$ point $\left(\approx 113^{\circ}\right)$. As the $\mathrm{CNN} / \mathrm{NNC}$ angles get small, the PESs of $S_{2}$ and $S_{1}$ approach (see Figure $2(\mathrm{e})$ ) and they almost cross at $\mathrm{CNN}=\mathrm{NNC}=100^{\circ}$. Due to the rigidity of the $2 \mathrm{D}$ scan, we do not observe a real degeneracy point, but still our results agree very well with those of a fully-relaxed CI optimization performed at a similar level of theory, ${ }^{11}$ that found a planar $S_{2} / S_{1}$ CI at CNN $=\mathrm{NNC}=105^{\circ}$. On the other hand, $S_{1}$ is much destabilized by small CNN/NNC values, and from the previously mentioned $S_{2} / S_{1}$ CI the system is much likely to follow the steep gradient along the symmetric bending coordinate, moving towards the planar $S_{1} / S_{0}$ CI-bend1.

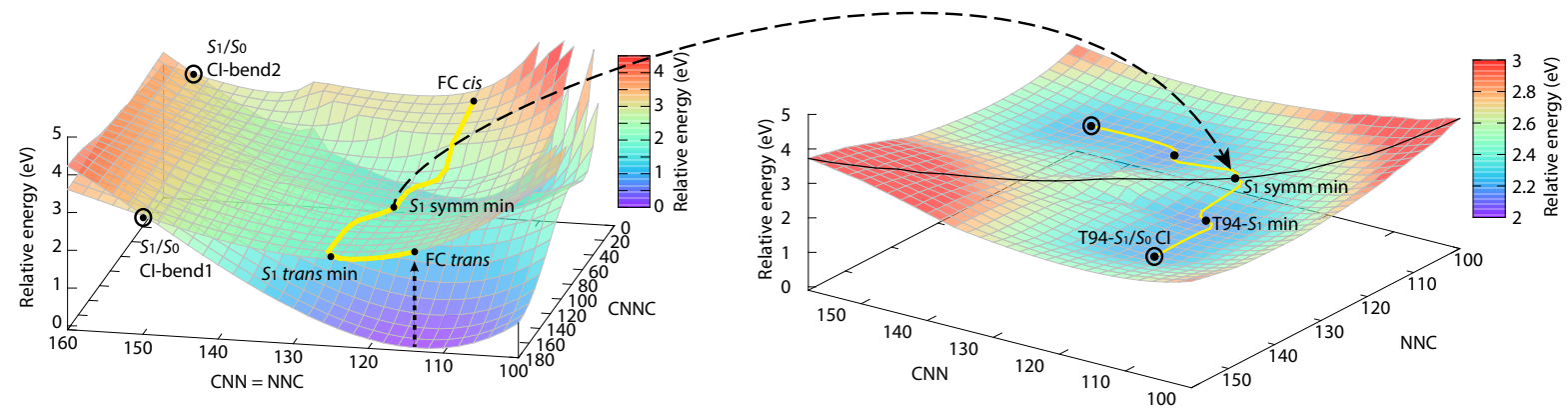

Figure 3: Representation of the MEP on $S_{1}$ (yellow line): on the left, in the space of CNNC torsion and symmetric CNN bending from FC point to $S_{1}$ symmetric minimum, on the right in the space of asymmetric bending at $\mathrm{CNNC}=94^{\circ}$, from $S_{1}$ symmetric minimum to the $S_{1}$ asymmetric minimum (T94- $S_{1}$ min) and lowest-energy CI (T94- $\left.S_{1} / S_{0} \mathrm{CI}\right)$.

\subsection{D surfaces in space of symmetric and asymmetric bending}

The first two rows of Figure 4 show the ground and $n \pi^{*}$ state PESs obtained with the 2D scans in the space of CNN and NNC bending angles as independent coordinates, for selected CNNC torsion values. The central part of Table 1 collects the geometrical parameters and energies of the relative minima (see supporting information for a full report of the CNN-NNC scans and details on the minima).

The ground-state minimum lies on the diagonal for all the CNNC torsional values (i.e., symmetric structure), and at $\mathrm{CNNC}=180^{\circ}$ it coincides with the $S_{0}$ trans min from the pre- 


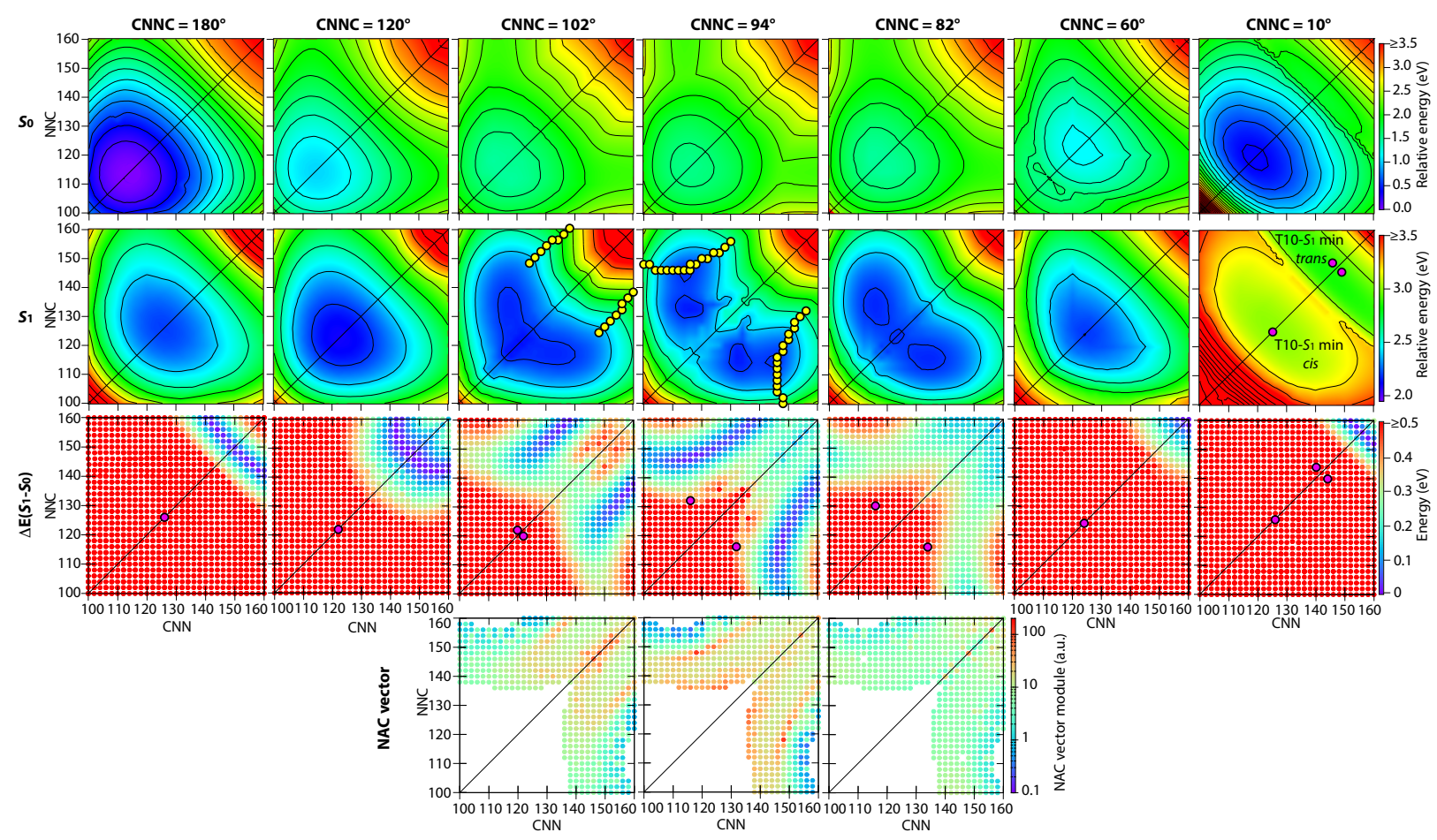

Figure 4: Top views of $S_{0}$ and $S_{1}$ PESs (isolines every $0.1 \mathrm{eV}$ ) and energy gap $E_{S_{1}-S_{0}}$ in the space of CNN and NCC bending angles for fixed torsion $180^{\circ}, 120^{\circ}, 102^{\circ}, 94^{\circ}, 82^{\circ}, 60^{\circ}$ and $10^{\circ}$ at the RASPT2 level (see computational methods); the diagonal connects symmetric structures. The yellow dots label the CIs whose energy is lower than the FC point, the purple dots indicate $S_{1}$ minima. For $102^{\circ}, 94^{\circ}$ and $82^{\circ}$ torsion the NAC vector module (in atomic units) is also displayed, for the points where $E_{S_{1}-S_{0}} \leqslant 0.3 \mathrm{eV}$. 
vious torsion/symmetric bending scan (see Table 1). On the other hand, the $n \pi^{*}$ minimum for the single scans has a symmetric geometry below $82^{\circ}$ and above $102^{\circ}$ torsion, while for $82^{\circ} \leq \mathrm{CNNC} \leq 102^{\circ}$ the PES shows two off-diagonal minima, even though the stabilization brought by the asymmetric deformation is not large $(\approx 0.2 \mathrm{eV}$ lower than the closest symmetric structures). Indeed, the $n \pi^{*}$ surface in our reduced space is extremely flat, and it is difficult to locate the absolute $S_{1}$ minimum in the $3 \mathrm{D}$ grid, since for $30^{\circ} \leq \mathrm{CNNC} \leq 150^{\circ}$ all the local $S_{1}$ minima are almost isoenergetic, or their energy difference falls below the RASPT2 error.

The third row of Figure 4 shows the $S_{1}-S_{0}$ energy gap. Far from $90^{\circ}$ torsion the crossing seam (violet region) has the shape of a straight line perpendicular to the diagonal, meaning that the crossing points are characterized by a constant value of the sum $\mathrm{CNN}+\mathrm{NNC}$ (that is always between $300^{\circ}$ and $315^{\circ}$ ). This is due to the fact that opening one of the two angles increases the repulsion with the respective nitrogen lone pair, requiring a reduction of the other angle to compensate and preserve degeneracy. However, for this torsion values, all the CIs are difficult to access with direct $n \pi^{*}$ excitation, since their energy is higher than the FC point $\left(E_{S_{0}}=E_{S_{1}}>3.0 \mathrm{eV}\right)$, and they lie far from the $S_{1}$ minima.

On the other hand, for $82^{\circ} \leqslant \mathrm{CNNC} \leqslant 102^{\circ}$ the crossing seam is much wider and includes only asymmetric structures, which is a common feature of many conjugated compounds. ${ }^{30}$ Indeed, the lowest-energy CIs of all the 3D grid are found between $90^{\circ}$ and $98^{\circ}$ torsion: we have found three isoenergetic crossing points, which are asymmetric and characterized by similar CNN-NNC bending values (labelled T98-, T94- and T90- $S_{1} / S_{0}$ CI in Table 1 ). Although these results are coming from a rigid scan, they perfectly match those of some fully-unconstrained CASPT2 optimizations, ${ }^{12,13}$ both in terms of energy and geometry. It is important to stress the fact that the surface of the $n \pi^{*}$ state in this region is so flat that the lowest points of the seam are almost isoenergetic with the corresponding $S_{1}$ minima (labelled T98-, T94- and T90-S $S_{1}$ min in Table 1), and the path connecting them is practically barrierless. The right part of Figure 3 shows the MEP on $S_{1}$ in the space of asymmetric CNN 
and $\mathrm{NNC}$ bending at $\mathrm{CNNC}=94^{\circ}$ : after moving along symmetric bending and torsion up to $S_{1}$ symm min, the molecule starts to desymmetrize due to the gradient along asymmetric bending, which drives it to the asymmetric $S_{1}$ minimum and lowest energy $S_{1} / S_{0}$ crossing point.

\subsection{MS-RASPT2 unconstrained optimizations}

The surface of $S_{1}$ in the 3D rigid scan is rather flat, therefore it is difficult to identify the absolute minimum and the lowest $S_{1} / S_{0}$ crossing point, since the energy difference with the nearby points of the grid often falls below the RASPT2 error. However, since we know that the absolute minimum on the $n \pi^{*}$ state has a twisted geometry (i.e., with a value of the CNNC dihedral between $100^{\circ}$ and $90^{\circ}$ ), and since in this region $S_{1}$ is stabilized by the desymmetrization of the two CNN/NNC bending angles, the asymmetric structure T94$S_{1}$ min (Figure $5(\mathrm{a})$ ) is a good model geometry for the absolute minimum from our 3D grid. Similarly, the structure T94- $S_{1} / S_{0}$ CI (Figure $5(\mathrm{~b})$ ) is the best approximation for the lowest $S_{1} / S_{0}$ CI from the rigid scan. This crossing point is also a local minimum on the excited state, separated from T94- $S_{1}$ min just by a very small barrier. Figure 6 shows a cross-section of the RASSCF, SS-RASPT2 and MS-RASPT2 potential energy surfaces at $94^{\circ}$ torsion including both structures. T94- $S_{1}$ min corresponds to the point with the minimum energy gap at RASSCF level $\left(\Delta E_{S_{1}-S_{0}}^{\mathrm{RASSCF}}=0.18 \mathrm{eV}\right)$, and in this point the two states are almost crossing also at the SS-RASPT2 level $\left(\Delta E_{S_{1}-S_{0}}^{\mathrm{SS}-\mathrm{RASP} 2}=0.11 \mathrm{eV}\right)$. However, after the wavefunction correction at MS-RASPT2 level, the two states split $\left(\Delta E_{S_{1}-S_{0}}^{\mathrm{MS} \text { ASPT2 }}=0.42 \mathrm{eV}\right)$. On the other hand, for the structure T94- $S_{1} / S_{0}$ CI, the RASSCF states are well separated in energy, and the SS- and MS-RASPT2 results are similar, resulting in a crossing point in both cases $\left(\Delta E_{S_{1}-S_{0}}^{\mathrm{SS}-\mathrm{RASPT} 2}=\Delta E_{S_{1}-S_{0}}^{\mathrm{MS} \text { RASPT2 }}=0.03 \mathrm{eV}\right)$. It is therefore possible that T94- $S_{1}$ min is an artifact of the RASPT2 correction, that suffers from the wavefunction mixing at RASSCF level, and that the actual excited state minimum corresponds to the crossing point T94- $S_{1} / S_{0}$ CI. 


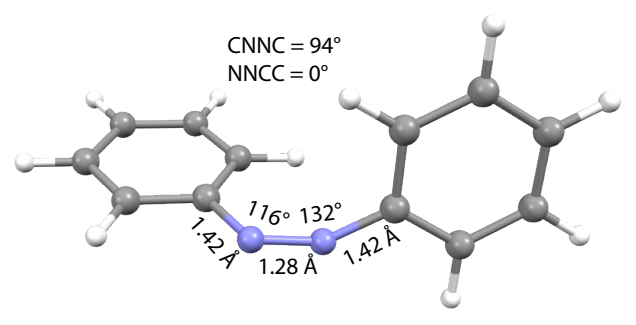

(a) $\mathrm{T} 94-S_{1} \min$

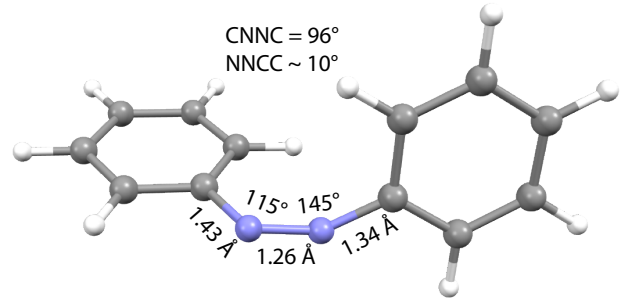

(c) optimized $S_{1}$ minimum

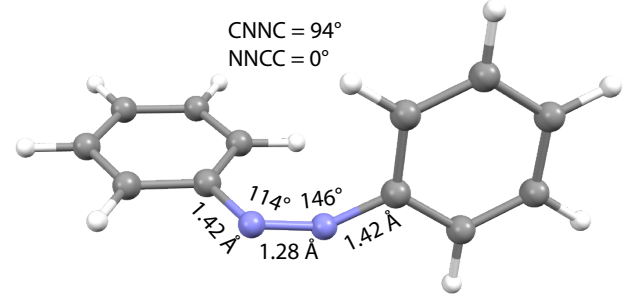

(b) $\mathrm{T} 94-S_{1} / S_{0} \mathrm{CI}$

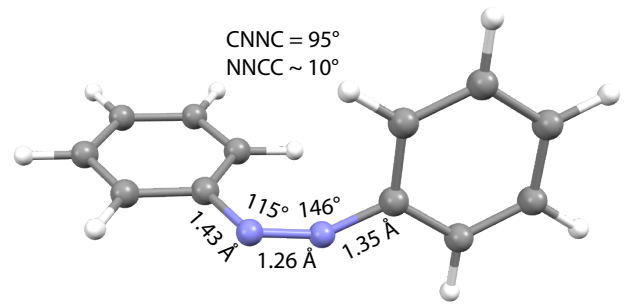

(d) optimized $S_{1} / S_{0} \mathrm{CI}$

Figure 5: Structures of the $S_{1}$ asymmetric minimum (a) and $S_{1} / S_{0}$ lowest-energy CI (b) from the rigid scan; optimized $S_{1}$ minimum (c) and $S_{1} / S_{0}$ CI (d).

In any case, in this region of small $S_{1}-S_{0}$ energy gap, the MS-RASPT2 correction gives a more balanced description, and we have used it for subsequent unconstrained optimizations. We performed a geometry optimization on the $n \pi^{*}$ state and a $S_{1} / S_{0}$ conical intersection optimization at the same level of theory of the rigid scan (MS-2-RASPT2/SA-2$\operatorname{RASSCF}(4,9|0,0| 4,7) /$ ANO-L-VDZP), both without constraints and without symmetry. To our knowledge, there are no other examples in literature of such accurate optimizations for azobenzene, employing such a wide active space. Figure 5 (c) and (d) show the resulting
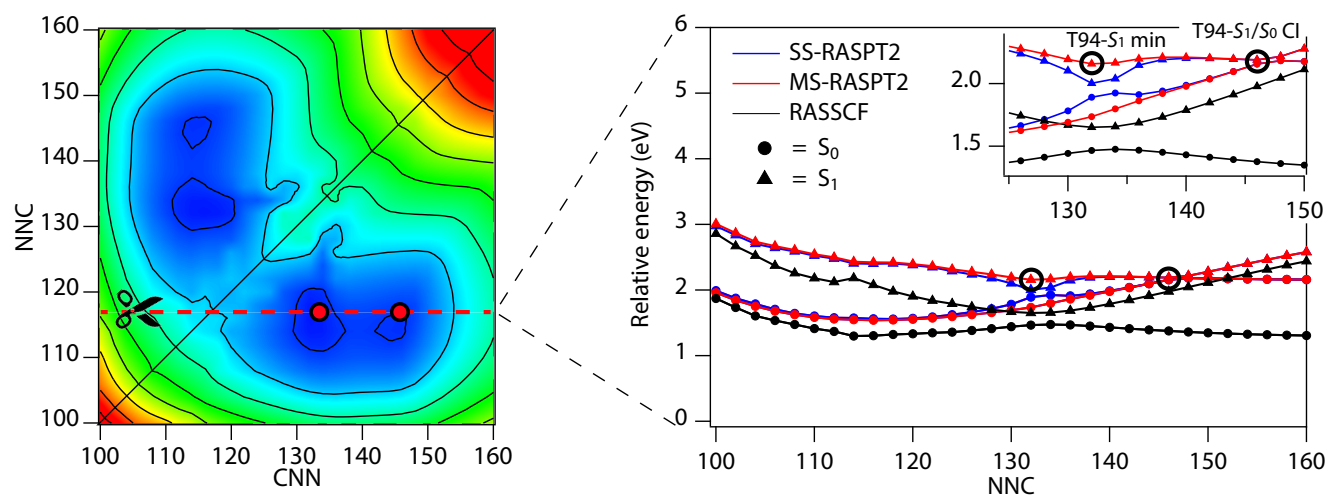

Figure 6: Cut through the $\mathrm{CNN} / \mathrm{NNC}$ bending PESs for $\mathrm{CNNC}=94^{\circ}\left(\right.$ at $\mathrm{CNN}=116^{\circ}$ ) showing both the T94- $S_{1}$ min and the lowest crossing point T94- $S_{1} / S_{0}$ CI. 
optimized structures, while the relative energies are reported in the lower part of Table 1.

The two structures are almost identical, confirming that the excited state minimum coincides with the lowest energy $S_{1} / S_{0}$ CI. Comparing this structure to the FC geometries, the most significant changes are along the CNNC torsion and CNN/NNC bending values, confirming that these coordinates are the most relevant ones both for the $n \pi^{*}$ relaxation and to reach the $S_{1} / S_{0}$ crossing seam. It is remarkable that the structure is asymmetric, and that the rigid scan predicts well the values of the CNNC dihedral and CNN/NNC bendings. In addition, the relative energy of the optimized $\mathrm{CI}$ is only $0.2 \mathrm{eV}$ lower than that of the CIs from the rigid scan, which means that other internal coordinates are only of secondary importance.

In a previous work, Conti et al. have performed some CI optimizations at the CASSCF level, using a 12 orbitals/14 electrons active space, and then recomputed the energy of the located minima at CASPT2 level. ${ }^{13}$ More recently, Casellas et al. located two $S_{1} / S_{0}$ CIs at CASPT2 level by means of grid calculations in the neighbourhood of some CASSCF optimized CIs, using a 8 orbitals/10 electrons active space. ${ }^{12}$ The most relevant geometrical parameters of the reported CIs can be found in Table 2, together with their energy, that we have recomputed at our level of theory.

In reference, ${ }^{13}$ the CIs were optimized at CASSCF level, and none of the reported geometries was found to be a real crossing point at CASPT2 level. Also recomputing the energy at our level of theory, we have found a non negligible gap between $S_{0}$ and $S_{1}$ for all the reported structures. However, for some of the twisted CIs (namely $\mathrm{CI}_{\text {tors }-1}$ and $\mathrm{CI}_{\text {tors }-2}$, see Table 2) the energy gap is not large $(<0.3 \mathrm{eV})$, confirming the presence of some crossing points nearby, as we have found with our rigid scan and unconstrained CI optimization.

Concerning the CIs reported in reference, ${ }^{12}$ the Planar CI-ref ${ }^{12}$ structure is a crossing point also at our level of theory, and is similar to our $S_{1} / S_{0}$ CI-bend1 geometry (Table 1), while recomputing the energies of Rotated CI-ref ${ }^{12}$ we have found a $S_{1}-S_{0}$ energy gap of $0.35 \mathrm{eV}$. 
Table 2: Structures and energies of $S_{1} / S_{0}$ crossing points from past literature, ${ }^{12,13}$ recomputed at the level of theory of our MS-RASPT2 optimizations.

\begin{tabular}{lcccccc|ccc}
\hline & & & \multicolumn{4}{c}{ literature energy $(\mathrm{eV})$} & \multicolumn{3}{c}{ recalculated energy (eV) } \\
& $\mathrm{CNNC}\left(^{\circ}\right)$ & $\mathrm{CNN}\left(^{\circ}\right)$ & $\mathrm{NNC}\left(^{\circ}\right)$ & $E_{S_{0}}$ & $E_{S_{1}}$ & $\Delta E_{S_{1}-S_{0}}$ & $E_{S_{0}}$ & $E_{S_{1}}$ & $\Delta E_{S_{1}-S_{0}}$ \\
\hline$E-\mathrm{CI}_{d-i n v}{ }^{13}$ & 180 & 157 & 157 & 3.08 & 3.71 & 0.36 & 3.26 & 4.05 & 0.79 \\
$Z-\mathrm{CI}_{d-i n v}{ }^{13}$ & 1 & 158 & 151 & 2.38 & 2.85 & 0.47 & 3.11 & 3.59 & 0.48 \\
$\mathrm{CI}_{\text {tors }-1}{ }^{13}$ & 94 & 136 & 117 & 2.06 & 2.26 & 0.20 & 2.15 & 2.42 & 0.27 \\
$\mathrm{CI}_{\text {tors }-2}{ }^{13}$ & 84 & 133 & 110 & 1.96 & 2.21 & 0.25 & 2.11 & 2.32 & 0.21 \\
$\mathrm{CI}_{\text {tors }-3}{ }^{13}$ & 92 & 139 & 119 & 2.24 & 2.55 & 0.31 & 2.22 & 2.59 & 0.37 \\
\hline Planar CI-ref $^{12}$ & 180 & 146 & 146 & 3.01 & 3.05 & 0.04 & 2.52 & 2.58 & 0.06 \\
Rotated CI-ref $^{12}$ & 93 & 138 & 118 & 2.19 & 2.23 & 0.04 & 1.81 & 2.16 & 0.35 \\
\hline
\end{tabular}

Even though the scenario from both references is in good agreement with our results, the fact that only one of the reported CIs is a real crossing point also at our level of theory demonstrates how strongly the electronic structure calculations are affected by the composition of the active space. The effect of the active space size is also visible in the absolute energy of the states: this is particularly evident in the case of the CIs reported by Casellas et al., ${ }^{12}$ for which the recomputed energies are about $0.5 \mathrm{eV}$ lower than the reported values.

\subsection{Non-adiabatic couplings}

In the previous section we have shown that the lowest crossing point between the $n \pi^{*}$ state and the ground state is found at $\mathrm{CNNC}=95^{\circ}$. Indeed, between $80^{\circ}$ and $100^{\circ}$ torsion we have the lowest energy gaps and the two states mix heavily. Wavefunction mixing in this region suggests that there is a finite $S_{1} \rightarrow S_{0}$ decay probability even in the points where the two states are not perfectly degenerate, and this can be quantified by the NAC vector.

We have computed the NAC vector in the subspace of the central CNNC atoms between $82^{\circ}$ and $102^{\circ}$ torsion (see computational methods); the results for $\mathrm{CNNC}=82^{\circ}, 94^{\circ}$ and $102^{\circ}$ are reported in the lowest part of Figure 4 . The NAC vector has largest magnitude for $94^{\circ}$ torsion, which is in agreement with the CI optimization and with evidence from the rigid scan. In addition, the fact that the highest values for the NACs are found for asymmetric bending geometries (i.e., close to the crossing seam) confirms the importance of 
Table 3: trans $\rightarrow$ cis photoisomerization quantum yield $\left(\Phi_{t \rightarrow c}\right)$ and excited state lifetime of the $n \pi^{*}$ state $\left(\tau_{\mathrm{n} \pi^{*}}\right)$ from our simulations and from literature data. $\left({ }^{(a)}=\right.$ extrapolated from ref. ${ }^{31}$ FMS = Full Multiple Spawning; TSH = Tully Surface Hopping; MD = Molecular Dynamics).

\begin{tabular}{ccc}
\hline & $\Phi_{t \rightarrow c}$ & $\tau_{\mathrm{n} \pi^{*}}(\mathrm{ps})$ \\
\hline This work, 300 K & 0.44 & 1.5 \\
FMS, ${ }^{31} 298 \mathrm{~K}$ & 0.46 & $0.4^{(a)}$ \\
TSH, ${ }^{31} 298 \mathrm{~K}$ & 0.33 & $0.3^{(a)}$ \\
MD $^{17} 300 \mathrm{~K}$ & 0.52 & 0.44 \\
\hline
\end{tabular}

desymmetrization for coupling the two states. It is worth noticing that the NAC values are not symmetric around the $\mathrm{CI}$ at $94^{\circ}$ torsion: indeed, the values found at $\mathrm{CNNC}=102^{\circ}$ are higher than those at $86^{\circ}$ (see Figure 4 and supporting information); this is a consequence of the fact that the crossing seam is wider on the "trans" side of the PES, which is one of the causes of the lower photoisomerization quantum yield starting from the trans isomer.

\subsection{Semi-classical dynamic simulations}

To asses the accuracy of our PESs, we fitted them to simulate the trans $\rightarrow$ cis photoisomerization at $300 \mathrm{~K}$ as described in the computational methods. Figure 7 shows the average values of the CNNC and CNN angles along the bunch of trajectories as well as the $S_{1}$ population as a function of time.

On average, the CNNC dihedral takes about $0.4 \mathrm{ps}$ to reach $90^{\circ}$. During this time, all the trajectories display in-phase, large amplitude oscillations of the CNN and NNC bending angles for 2-3 periods. It is also worth noting that due to the rigidity of the system, the combined effect of the flatness of the $S_{1}$ PES along the CNNC torsion coordinate and the initial momentum along torsion of system make the molecule reach $\mathrm{CNNC}$ values up to $40^{\circ}$ already in this short period of time. As soon as the trajectory approaches $90^{\circ}$ torsion, the two CNN angles desymmetrize, since the $n \pi^{*} \mathrm{PES}$ in this region is characterized by asymmetric minima. Furthermore, the oscillation amplitude of the CNN angles decreases, due to an energy transfer from the bending to the torsional mode.

In Table 3 the obtained excited state lifetime $\left(\tau_{\mathrm{n} \pi^{*}}\right)$ and photoisomerization quantum 


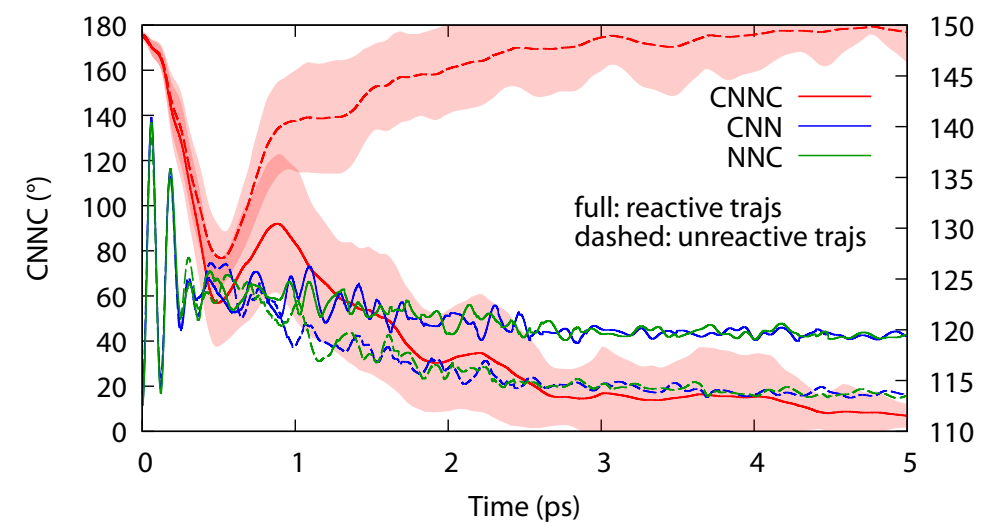

0
$u$
$z$
$z$
0
$\frac{1}{0}$
$z$
$z$

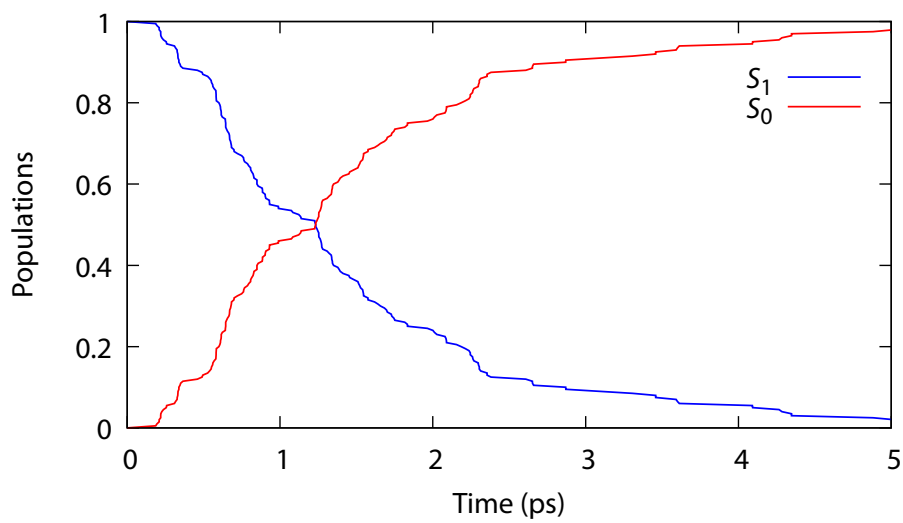

Figure 7: Average values of the CNNC and CNN angles along the bunch of trajectories, with $\pm 1 / 2$ standard deviation interval represented as shaded area (top) and $S_{1}$ and $S_{0}$ populations as a function of time (bottom).

yield $\left(\Phi_{t \rightarrow c}\right)$ are compared with literature data. The obtained $\Phi_{t \rightarrow c}$ is 0.44 , which is in good agreement with the results of past simulations. ${ }^{17,31}$ On the other hand, the resulting $S_{1}$ lifetime is almost three times larger than literature data. ${ }^{17,31}$ Indeed, even if the time required to reach twisted geometries is perfectly in line with past simulations, ${ }^{15}$ the average decay time is longer, resulting a $\tau_{\mathrm{n} \pi^{*}}$ of $1.5 \mathrm{ps}$. This is likely to be a consequence of two main approximations: first of all, the molecule is allowed to decay only in the $82^{\circ} \leq \mathrm{CNNC} \leq 102^{\circ}$ window, where we have mapped the NAC. Secondly, we must remember that the PES of the excited state was calculated with a rigid scan starting from the trans ground state minimum, thus the $S_{1}-S_{0}$ energy gap is overestimated, with the effect of reducing the hopping probability. In addition, even though the frozen degrees of freedom are not fundamental for the description of the photoprocess, they can act as a thermal bath, influencing the molecular motion and the atomic velocities in a way that goes beyond the simple scaling factor that 
we have introduced in the calculation of the decay probability (see computational methods).

\section{Summary and outlook}

In this work we have used accurate ab initio calculations (MS-2-RASPT2/SA-2-RASSCF $(4,9|0,0| 4,7) /$ ANO-L-VDZP) to map the PESs of the ground and first $n \pi^{*}$ excited state of azobenzene along the most relevant degrees of freedom for coupling the two states (i.e., CNNC torsion and the two CNN bending angles). Together with the energies, we have provided a broad map of the non-adiabatic coupling between the two states in the region where they get closer in energy. To our knowledge, such an accurate and extended mapping of the PESs of azobenzene has never been reported before. Our data provide an unprecedented look at the topology of the CI seam between the $n \pi^{*}$ state and the ground state and prove the importance of desymmetrization to efficiently couple the electronic states and reach the low energy region of the crossing seam. Indeed, the intersection space includes both symmetric and asymmetric structures: the former lie at high energy and are more accessible when exciting the $\pi \pi^{*}$ state (as predicted in a recent work ${ }^{11}$ ), since they are reached mainly through the symmetric bending coordinate. In contrast, dynamics initiated in the $n \pi^{*}$ state could decay predominantly through torsion, which leads to the lowest points of the CI seam, characterized by twisted geometries $\left(\mathrm{CNNC} \approx 90^{\circ}\right)$, asymmetric bending and higher photoisomerization quantum yield.

Scanning CNNC torsion and CNN/NNC bending has proven to be sufficient to correctly describe the topology of the PESs as well as to catch the most relevant CIs, as confirmed by comparison with fully unconstrained optimizations at the same level of theory. The accuracy of our PESs was further validated with simplified semiclassical dynamics simulations in the reduced space of the three scanned coordinates, and the results are in good agreement with past simulations. This is true especially for the photoisomerization quantum yield and the geometries explored by the excited state trajectories, while the resulting $n \pi^{*}$ lifetime 
is longer than in previous studies: ${ }^{17,31}$ further dynamics simulations may therefore reveal wether this is a consequence of our approximations or actually previous simulations suffered from inaccurate description of the CI region.

Further improvements include extending the NAC calculations to a larger region, especially between $90^{\circ}$ and $110^{\circ}$ torsion, thus providing a wider crossing seam region for the decay.

This work also provides the needed ingredients to perform subsequent more accurate dynamics, either fully-quantum dynamics along the reactive coordinates or classical and semiclassical MD along all the degrees of freedom, employing a suitable parameterized potential. Even though examples of both classical and semi-classical MD simulations using ad hoc parameterizations (i.e., force fields ${ }^{17}$ or semiempirical Hamiltonian ${ }^{14}$ ) have already been reported in the literature, our calculations could further improve the quality of the simulations, due to the accuracy of the QM methods used for the scan. In particular, our 3D scan for the first time analyzes in detail the CI seam around $90^{\circ}$. Treating the two CNN bending angles as independent coordinates is essential to break the symmetry, as large non-adiabatic mixing (and thus effective decay) occurs at large asymmetries for the CNN bendings (e.g., $\left.116^{\circ} / 146^{\circ}\right)$.

\section{Acknowledgement}

Marco Garavelli acknowledges support from the H2020-NMBP-TO-IND-2018-2020/DT-NMBP09-2018 project SIMDOME, Grant agreement No. 814492.

Claudio Zannoni would like to thank the Isaac Newton Institute for Mathematical Sciences, Cambridge, UK, for support and hospitality during the program Mathematical Design of New Materials when work on this paper was completed. This work was supported by an EPSRC Grant. 


\section{Supporting Information Available}

A graphical representation of the active space orbitals is provided in the supporting information. Also, we compare the SS-RASPT2 and MS-RASPT2 results, motivating our choices. Then we provide the PESs obtained with the two separate rigid scans (i.e., starting from trans or cis ground state minimum) involving CNNC torsion and symmetric CNN/NNC bending. Data from the asymmetric bending scans at $\mathrm{CNNC}=150^{\circ}, 98^{\circ}, 90^{\circ}, 86^{\circ}$ and $30^{\circ}$ (omitted from main text) are also reported, as well as NAC data, where calculated. We also report the cartesian coordinates of the optimized $S_{1}$ minimum and $S_{1} / S_{0}$ optimized CI, and a graphical representation of the full-coordinates NAC vector calculated for benchmarking. Finally, we report detail about the PESs fitting used for semiclassical dynamics and the scaling factor used for the atomic velocities.

This material is available free of charge via the Internet at http://pubs.acs.org/.

\section{References}

(1) Guin, T.; Settle, M. J.; Kowalski, B. A.; Auguste, A. D.; Beblo, R. V.; Reich, G. W.; White, T. J. Layered liquid crystal elastomer actuators. Nat. Commun. 2018, 9, 2531.

(2) Shang, Y.; Wang, J.; Ikeda, T.; Jiang, L. Bio-inspired liquid crystal actuator materials. J Mater Chem C 2019, 7, 3413-3428.

(3) Tonnelé, C.; Pershin, A.; Gali, S. M.; Lherbier, A.; Charlier, J.-C.; Castet, F.; Muccioli, L.; Beljonne, D. Atomistic simulations of charge transport in photoswitchable organic-graphene hybrids. Journal of Physics: Materials 2019, 2, 035001.

(4) Fregoni, J.; Granucci, G.; Coccia, E.; Persico, M.; Corni, S. Manipulating azobenzene photoisomerization through strong light-molecule coupling. Nat. Commun. 2018, 9, 4688. 
(5) Natansohn, A.; Rochon, P. Photoinduced Motions in Azobenzene-Based Amorphous Polymers: Possible Photonic Devices. Adv. Mater. 1999, 11, 1387-1391.

(6) Cao, Y.; Dong, S.; Liu, S.; Liu, Z.; Guo, X. Toward Functional Molecular Devices Based on GrapheneMolecule Junctions. Angew. Chem. Int. Ed. 2013, 52, 3906-3910.

(7) Meng, L.; Xin, N.; Hu, C.; Wang, J.; Gui, B.; Shi, J.; Wang, C.; Shen, C.; Zhang, G.; Guo, H.; Meng, S.; Guo, X. Side-group chemical gating via reversible optical and electric control in a single molecule transistor. Nat. Commun. 2019, 10, 1450.

(8) Emoto, A.; Uchida, E.; Fukuda, T. Optical and Physical Applications of Photocontrollable Materials: Azobenzene-Containing and Liquid Crystalline Polymers. Polymers $2012,4,150-186$.

(9) Mativetsky, J. M.; Pace, G.; Elbing, M.; Rampi, M. A.; Mayor, M.; Samor, P. Azobenzenes as Light-Controlled Molecular Electronic Switches in Nanoscale MetalMoleculeMetal Junctions. J. Am. Chem. Soc. 2008, 130, 9192-9193.

(10) Lednev, I.; Ye, T.-Q.; Matousek, P.; Towrie, M.; Foggi, P.; Neuwahl, F.; Umapathy, S.; Hester, R.; Moore, J. Femtosecond time-resolved UV-visible absorption spectroscopy of trans-azobenzene: dependence on excitation wavelength. Chem. Phys. Lett. 1998, 290, $68-74$.

(11) Nenov, A.; Borrego-Varillas, R.; Oriana, A.; Ganzer, L.; Segatta, F.; Conti, I.; SegarraMarti, J.; Omachi, J.; Dapor, M.; Taioli, S.; Manzoni, C.; Mukamel, S.; Cerullo, G.; Garavelli, M. UV-Light-Induced Vibrational Coherences: The Key to Understand Kasha Rule Violation in trans-Azobenzene. J. Phys. Chem. Lett. 2018, 9, 1534-1541.

(12) Casellas, J.; Bearpark, M. J.; Reguero, M. ExcitedState Decay in the Photoisomerisation of Azobenzene: A New Balance between Mechanisms. ChemPhysChem 2016, 17, 3068-3079. 
(13) Conti, I.; Garavelli, M.; Orlandi, G. The Different Photoisomerization Efficiency of Azobenzene in the Lowest np* and pp* Singlets: The Role of a Phantom State. J. Am. Chem. Soc. 2008, 130, 5216-5230.

(14) Cusati, T.; Granucci, G.; Martnez-Nez, E.; Martini, F.; Persico, M.; Vzquez, S. Semiempirical Hamiltonian for Simulation of Azobenzene Photochemistry. J. Phys. Chem. A 2012, 116, 98-110.

(15) Ciminelli, C.; Granucci, G.; Persico, M. The Photoisomerization Mechanism of Azobenzene: A Semiclassical Simulation of Nonadiabatic Dynamics. Chem. - Eur. J. 2004, 10, 2327-2341.

(16) Granucci, G.; Persico, M. Excited state dynamics with the direct trajectory surface hopping method: azobenzene and its derivatives as a case study. Theor. Chem. Acc. 2007, 117, 1131-1143.

(17) Tiberio, G.; Muccioli, L.; Berardi, R.; Zannoni, C. How Does the Trans-Cis Photoisomerization of Azobenzene Take Place in Organic Solvents? ChemPhysChem 2010, 11, $1018-1028$.

(18) Tully, J. C. Molecular dynamics with electronic transitions. J. Chem. Phys. 1990, 93, $1061-1071$.

(19) Ishikawa, T.; Noro, T.; Shoda, T. Theoretical study on the photoisomerization of azobenzene. J. Chem. Phys. 2001, 115, 7503-7512.

(20) Gagliardi, L.; Orlandi, G.; Bernardi, F.; Cembran, A.; Garavelli, M. A theoretical study of the lowest electronic states of azobenzene: the role of torsion coordinate in the cis-trans photoisomerization. Theor. Chem. Acc. 2004, 111, 363-372.

(21) Wei-Guang Diau, E. A New Trans-to-Cis Photoisomerization Mechanism of Azobenzene on the S1(n,p*) Surface. J. Phys. Chem. A 2004, 108, 950-956. 
(22) Widmark, P.-O.; Malmqvist, P.-A.; Roos, B. O. Density matrix averaged atomic natural orbital (ANO) basis sets for correlated molecular wave functions. Theor. Chim. Acta 1990, r7, 291-306.

(23) Aquilante, F.; Autschbach, J.; Carlson, R. K.; Chibotaru, L. F.; Delcey, M. G.; De Vico, L.; Fdez. Galvn, I.; Ferr, N.; Frutos, L. M.; Gagliardi, L.; Garavelli, M.; Giussani, A.; Hoyer, C. E.; Li Manni, G.; Lischka, H.; Ma, D.; Malmqvist, P. .; Mller, T.; Nenov, A.; Olivucci, M.; Pedersen, T. B.; Peng, D.; Plasser, F.; Pritchard, B.; Reiher, M.; Rivalta, I.; Schapiro, I.; Segarra-Mart, J.; Stenrup, M.; Truhlar, D. G.; Ungur, L.; Valentini, A.; Vancoillie, S.; Veryazov, V.; Vysotskiy, V. P.; Weingart, O.; Zapata, F.; Lindh, R. Molcas 8: New capabilities for multiconfigurational quantum chemical calculations across the periodic table. J. Comput. Chem. 2016, 37, 506-541.

(24) Weingart, O.; Nenov, A.; Altoè, P.; Rivalta, I.; Segarra-Martí, J.; Dokukina, I.; Garavelli, M. COBRAMM 2.0 - A software interface for tailoring molecular electronic structure calculations and running nanoscale (QM/MM) simulations. J. Mol. Model. 2018, 24, 271.

(25) Altoè, P.; Stenta, M.; Bottoni, A.; Garavelli, M. A tunable QM/MM approach to chemical reactivity, structure and physico-chemical properties prediction. Theor. Chem. Acc. 2007, 118, 219-240.

(26) Phillips, J. C.; Braun, R.; Wang, W.; Gumbart, J.; Tajkhorshid, E.; Villa, E.; Chipot, C.; Skeel, R. D.; Kal, L.; Schulten, K. Scalable molecular dynamics with NAMD. J. Comput. Chem. 2005, 26, 1781-1802.

(27) Vetrkov, .; Ladnyi, V.; Al Anshori, J.; Dvok, P.; Wirz, J.; Heger, D. The absorption spectrum of cis-azobenzene. Photochem. Photobiol. Sci. 2017, 16, 1749-1756.

(28) Klessinger, M.; Michl, J. Excited states and photochemistry of organic molecules; WileyVCH, 1995. 
(29) Tan, E. M. M.; Amirjalayer, S.; Smolarek, S.; Vdovin, A.; Zerbetto, F.; Buma, W. J. Fast photodynamics of azobenzene probed by scanning excited-state potential energy surfaces using slow spectroscopy. Nat. Commun. 2015, 6, 5860.

(30) Nenov, A.; de Vivie-Riedle, R. Geometrical and substituent effects in conical intersections: Linking chemical structure and photoreactivity in polyenes. J. Chem. Phys. 2011, 135, 034304.

(31) Toniolo, A.; Ciminelli, C.; Persico, M.; Martnez, T. J. Simulation of the photodynamics of azobenzene on its first excited state: Comparison of full multiple spawning and surface hopping treatments. J. Chem. Phys. 2005, 123, 234308. 


\section{Graphical TOC Entry}

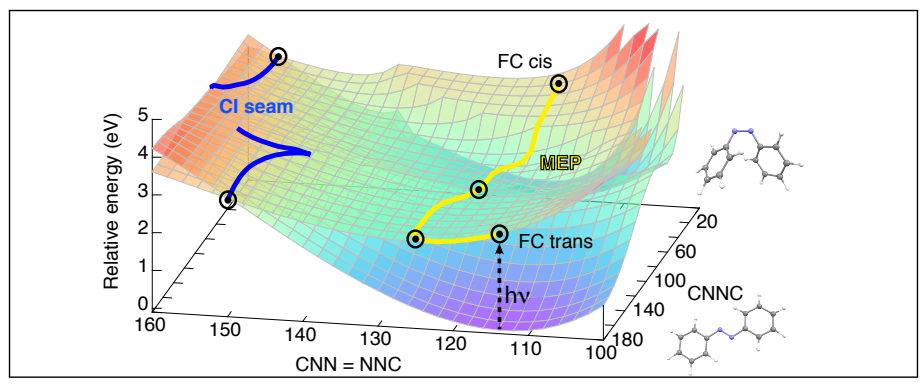

